\title{
Patient-held medical records for patients with chronic disease: a systematic review
}

\author{
Henry Ko, ${ }^{1}$ Tari Turner, ${ }^{1}$ Christine Jones, ${ }^{2}$ Caron Hill ${ }^{2}$
}

${ }^{1}$ Centre for Clinical Effectiveness, Southern Health, Clayton, Australia

${ }^{2}$ Acute Ambulatory Services, Southern Health, Clayton, Australia

\section{Correspondence to}

Dr Henry Ko, Centre for Clinical Effectiveness, Southern Health, 43-51 Kanooka Grove, Clayton, VIC 3168, Australia; henry.ko@med.monash.edu.au

Accepted 20 February 2010 Published Online First 28 May 2010
ABSTRACT

Objectives To determine whether in patients with chronic disease a patient-held medical record (PHR), compared to usual care, improves clinical care, patient outcomes or satisfaction.

Design Systematic review.

Data sources Databases searched were All EBM (The Cochrane Database of Systematic Reviews, DARE CENTRAL), Medline, CINAHL and EMBASE from 1980 to 16 February 2009.

Study selection Two reviewers assessed comparative studies that compared paper-based PHR to usual care for inclusion using a priori study selection criteria.

Studies reviewed Four hundred and eighty-one articles were reviewed by title and abstract. Full text was retrieved for 120 articles. Fourteen studies met the inclusion and exclusion criteria and were appraised using a priori criteria for methodological quality.

Results Fourteen studies were included in diabetes, oncology, mental health, rheumatoid arthritis, stroke and palliative care. The studies used a variety of designs of PHR and compared this with usual care. PHR were implemented with varying degrees of patient and staff support and education, mainly for six months or less. Outcomes included attitudes on the usefulness of PHR, the quality of information exchange, process indicators, and clinical and physiological indicators. The effectiveness of PHRs is generally of low or very low quality, with the majority of studies having a high risk of bias. These studies do not demonstrate a significant benefit of introducing PHR.

Conclusions There is no clear benefit of implementing a PHR, and due to medium to high risk of bias these findings should be interpreted with caution. More high quality studies are needed to evaluate properly the effectiveness of PHRs in chronic disease populations.

\section{INTRODUCTION}

In most care settings, the patient's medical or health record is held by the health service that is providing care to the patient. There has been interest in the concept of 'patient-held' medical records (PHR), where the patient is given a copy of the record to keep, and to take to health appointments, to help manage healthcare tasks and communication. PHRs are formal and structured records that are given to patients to enable the continuity and quality of care. ${ }^{1}$ They are also known as 'logbooks,' 'patient travelling records' or 'shared care diaries,' and include sections containing key patient and healthcare information, and usually contain blank sections to enable patient note-taking and healthcare staff notes. The suggestion that PHRs may be useful for patients with chronic disease comes from the use of PHRs in other healthcare areas such as in paediatrics, ${ }^{2-4}$ family health ${ }^{56}$ and community health. ${ }^{7}$ However, there are conflicting opinions on the effectiveness of PHRs, and they have been used with varying degrees of success.

\section{METHODS}

\section{Search strategy}

We performed a systematic search of the literature published in English language from 1980 onwards. Databases searched were All EBM (The Cochrane Database of Systematic Reviews, DARE, CENTRAL and ACP Journal Club), Medline (Ovid MEDLINE (R) In-Process \& Other Non-Indexed Citations, MEDLINE(R) and OLDMEDLINE(R) 1950 to Present), CINAHL (EBSCOhost), and EMBASE. These were searched from 1980 to 16 February 2009.

Our search in Medline was: (exp medical records/ or exp medical records, problem-oriented/or exp medical records systems, computerized/or exp nursing records/or (((medical or health) and

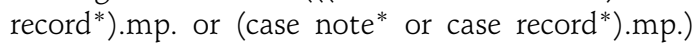
AND ((held adj3 (patient or parent or person or woman or man or family or consumer)).mp. or (carried adj3 (patient or parent or person or woman or man or family or consumer)).mp.)) OR ((logbook* or logbook*).mp. or passport*.mp. or personal health record*.mp.). Similar searches were used for the other databases.

\section{Study selection and inclusion criteria}

The selection criteria were defined before searching for studies. Inclusion criteria were any comparative study with:

- patients of any age with any chronic disease in any healthcare setting;

- an intervention in the form of a paper-based medical record held by the patient or their carer, with or without other interventions such as additional education for staff, reminder posters in clinics, and/or dedicated PHR coordinating staff;

- comparisons with no PHR (usual care) or 'sham' PHR, including usual medication lists, monitoring booklets (eg, for blood glucose monitoring), and care instructions given to patients; because patients may also use medicine lists or appointment booklets on their own initiative, we considered any unstructured booklet as a control treatment; any general notes made by the patient or unstructured records than the intervention were also classified as comparators. 
- outcomes comparing the effectiveness of the PHR on any measure relevant to patients, clinicians, or health services.

\section{Exclusion criteria were:}

- interventions such as advanced directives, which do not provide a detailed medical history of the patient, or

- electronic health records, including electronic health records that are controlled by the patient.

\section{Data extraction and quality assessment}

We included any studies reporting comparative data. For any unique population, intervention or comparative outcomes, systematic reviews and randomised controlled trials (RCT) were sought. In the absence of systematic reviews and RCTs, if there were unique populations, interventions or relevant outcomes addressed by lower-quality comparative studies (eg, cohort studies), then these were used.

Studies were selected and appraised independently by two reviewers in consultation with colleagues using study selection and standard appraisal criteria established a priori (see full appraisal report at http://www.southernhealth.org.au/page/ Health_Professionals/CCE/Evidence_reviews/). Studies were initially reviewed by title and abstract. When a decision on inclusion or exclusion could not be made based on title and abstract alone, full text was retrieved. Authors were contacted for further information where required.

\section{Data synthesis}

Given the diversity of chronic disease populations, the non-uniformity of intervention and control treatments, and variations in outcome measures, a statistical meta-analysis was not appropriate, so we undertook a narrative synthesis. Studies were grouped into chronic disease areas and the results interpreted in the context of their methodological strengths and weaknesses. Any contextual factors that might affect outcomes were also analysed.

\section{RESULTS}

Our search identified 481 articles. One hundred and twenty full text articles were retrieved for review, and 14 studies met the inclusion and exclusion criteria (figure 1).

Fourteen studies, represented by 15 articles, met our study selection criteria, and their characteristics are listed in table 1. Six populations are represented in these studies-diabetes, oncology, mental health, rheumatoid arthritis, stroke and palliative care. In diabetes, there were three cluster randomised controlled trials (cluster RCT), ${ }^{8-11}$ of which two articles were from the same study. ${ }^{89}$ In oncology, there were four RCTs, ${ }^{12-15}$ one crossover RCT $^{16}$ and one cohort study. ${ }^{17}$ In mental health, there were two cluster RCTs. ${ }^{18}{ }^{19}$ In rheumatoid arthritis, there was one controlled trial (CT) ${ }^{20}$ In stroke, there was a cohort study with a historical control. ${ }^{21}$ In palliative care, there was one RCT. $^{22}$

Appraisal of these articles indicates that the body of evidence is generally of low methodological quality. Table 2 shows that most studies do not meet key methodological criteria. Therefore, the results of the studies must be interpreted with caution. These weaknesses mean the potential effectiveness of the treatments may be exaggerated.

The results of these studies do not demonstrate that there is a significant benefit of introducing patient-held records. Some studies report small benefits or detriments on isolated measures, but most are not objective measures, may be biased by the design of the studies and are not consistent across studies. The

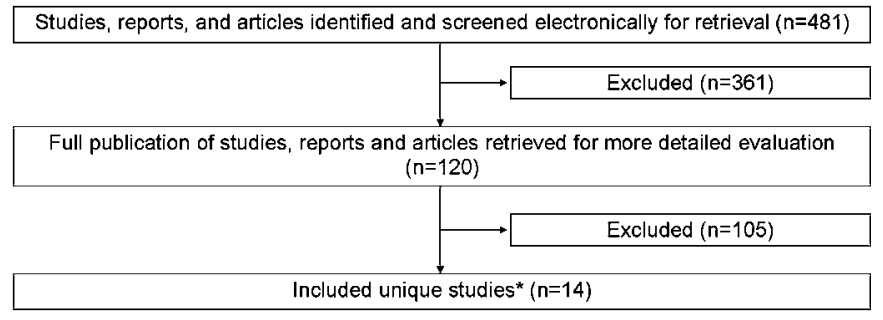

Figure 1 Flow diagram of included studies. *Two separate publications reported different outcomes from the same study. ${ }^{8}$

summary of the appraisals of study quality for each chronic disease group is presented in table 3 , and the individual studies are discussed below.

\section{Diabetes}

Three studies, represented by four articles, in diabetes as summarised in table 1 include a total of 3807 patients. All three studies have a moderate to high risk of bias. See table 2 for details about the methodological limitations.

Each of the studies reports a small number of benefits of PHRs. Dijkstra et $a l^{8}$ reported significant differences for five out of 17 outcomes. These include increases patients receiving foot examinations (OR: 1.68; 95\% CI 1.12 to 2.50 ), having physical exercise advised (OR: 1.84; 95\% CI 1.16 to 2.92), smoking discussed or non smoking advised (OR: 1.82; 95\% CI 1.15 to $2.89)$, decreases in HbA1c $(p<0.001)$ and a decrease in diastolic blood pressure $(\mathrm{p}<0.05)$. In Dijkstra et al ${ }^{10}$ there are differences in seven out of 21 outcome measures. There are benefits in the PHR group for process indicators of checking HbA1c (OR: 1.8; 95\% CI 1.2 to 2.7 ), creatinine (OR: $2.1 ; 95 \%$ CI 1.5 to 3.1 ), eye exam (OR: 1.7; 95\% CI 1.1 to 2.5$)$, cholesterol (OR: 1.9; 95\% CI 1.3 to 2.7 ), weight measured (OR: $2.2,95 \%$ CI 1.5 to 3.4 ) and glucose exam (OR: 1.6, 95\% CI 1.0 to 2.6 ) within certain time frames. ${ }^{10}$ Patients in the PHR group also have better knowledge of their own HbA1c level than control patients (OR: 1.7; 95\% CI 1.0 to 2.9 ). In Simmons et a ${ }^{11}$ there are differences in only two out of the 19 outcomes-relative reductions in HbA1c levels ( $\mathrm{p} 0.017)$ and increases in body mass index $(\mathrm{p}=0.028)$ favouring the PHR group. The other outcomes are not different between groups. As investigated by Dijkstra et al, ${ }^{9}$ even though life expectancy and quality-adjusted life years increased with PHR use, by 0.63 years and 0.59 , respectively, the economic outcomes presented suggest that any potential small benefit of PHRs in this population is not justified by the additional cost required to implement PHRs.

\section{Oncology}

Six studies of PHRs in oncology patients as summarised in table 1 include a total of 1773 patients. All six studies have a high risk of bias. See table 2 for detail about the methodological limitations.

Most outcomes measured by these studies showed no significant differences between groups, and no clear advantage of using a PHR. Cornbleet et al ${ }^{12}$ measured 11 opinions and attitudes of patients and staff and found no differences between treatments. There are few differences in outcomes in the other studies. Drury et $a l^{13}$ found that PHR users felt less able to face future aspects of their illness $(p=0.05)$, which was the only one significant result of 22 comparative measures. Finlay and Wyatt ${ }^{16}$ noted that PHR users used their record more than the control group $(p<0.001)$, and recorded medications more often $(p<0.001)$. However, control group patients who were using an 

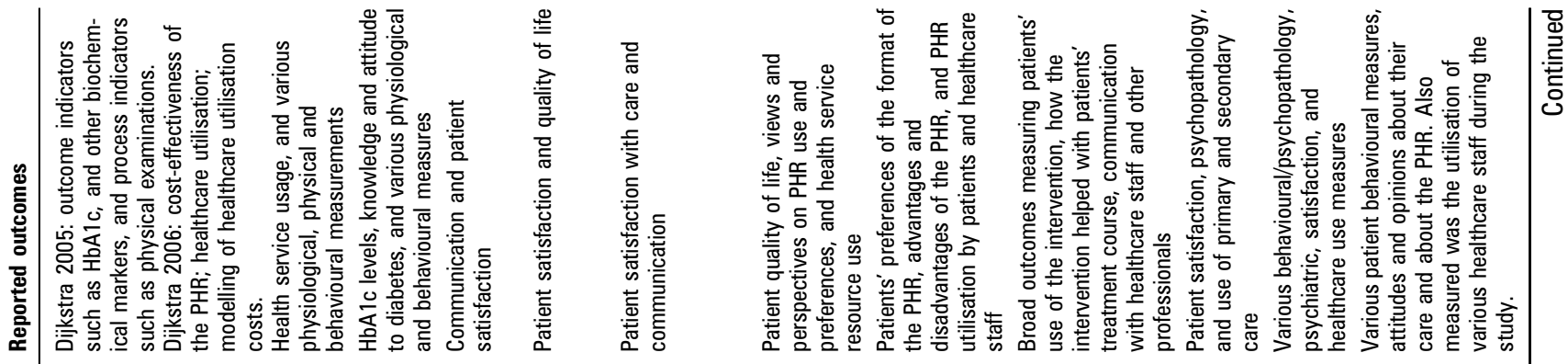

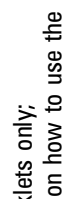

$\frac{\sqrt{3}}{\frac{0}{3}}$

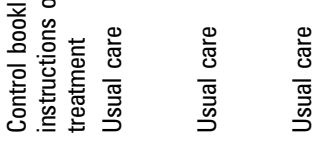

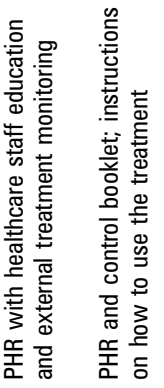

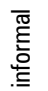

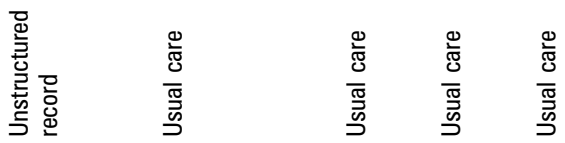

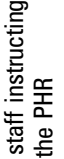

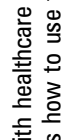

责点
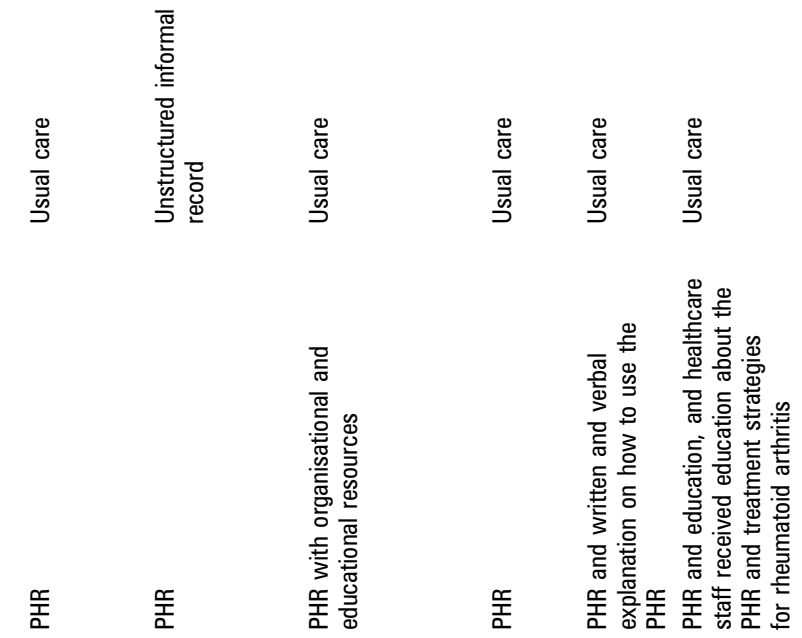

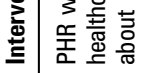

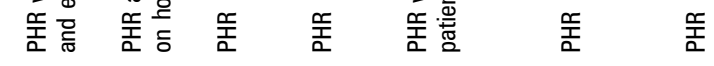
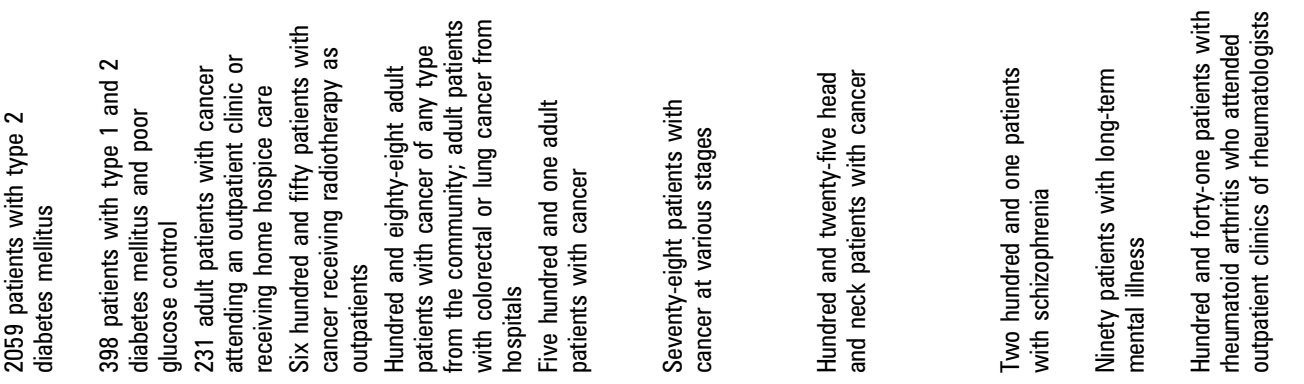

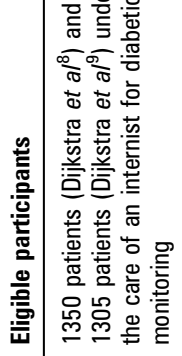

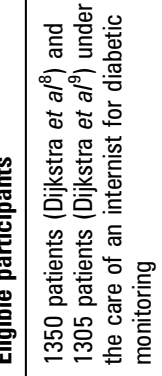

喜蒡

$\frac{\infty}{50} \frac{0}{5}$

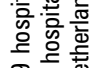

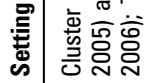

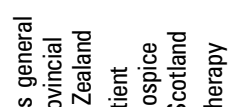

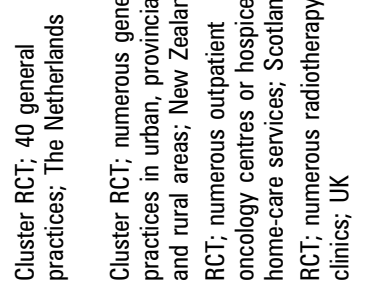

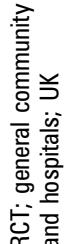

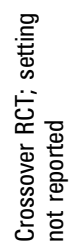

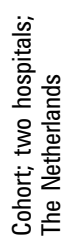

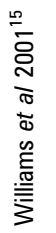

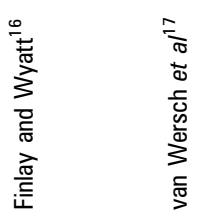

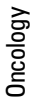

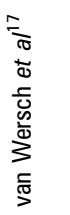

흥
등

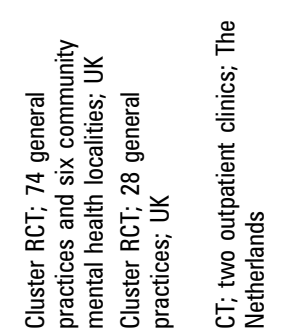

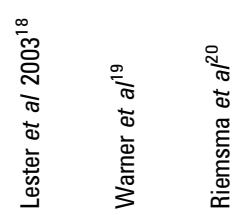

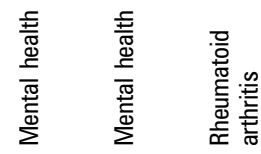




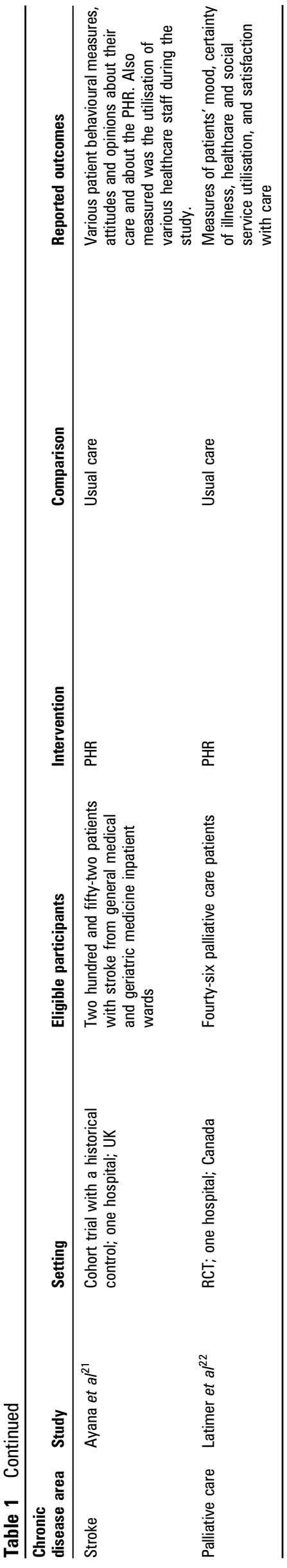

unstructured control record, had more questions $(p<0.008)$ and comments about their symptoms $(p<0.003)$ and saw more use by carers or relatives and healthcare staff $(p<0.02) .{ }^{16}$ Overall there are no differences for the majority of the 26 measures used in this study. In Lecouturier et al, ${ }^{14}$ only one out of eight comparative measures was different-that of patient satisfaction with information received at the end of treatment which favoured the control treatment $(\mathrm{p}=0.02)$. In the study by van Wersch et al, ${ }^{17}$ fewer PHR users compared with the control group felt they needed more information about their disease and treatment $(p \leq 0.05)$. However, the control group was less uncertain about tests, procedures and lifestyle issues $(p \leq 0.05) .{ }^{17}$ Support from various staff was similar between groups, but there was more support given to the intervention group from social nurses and social workers in coping with possible tension and other problems $(p<0.001) ;{ }^{17}$ this may be due to the nature of the intervention which included care from a social nurse. There were fewer patients in the intervention group reporting tension $(p=0.001)$, fear $(p=0.01)$, anxiety $(p=0.01)$ and depression $(p=0.01)$, but no differences for other psychosocial outcomes. ${ }^{17}$ The study by Williams et al ${ }^{15}$ shows no differences between the intervention and control group in most of the 15 quality of life subscales, 45 patient attitude/opinion measures and seven process measures. Patients using a PHR found it less difficult to monitor their own progress $(p=0.009)$ and were more likely to feel in control $(p=0.03) .{ }^{15}$

\section{Mental health}

Two studies assessing a PHR intervention in the mental health area are summarised in table 1 . They both have a high risk of bias. See table 2 for detail about the methodological limitations. These studies report no differences on any outcome measures. In the study by Lester et al ${ }^{18}$ there is no difference between the treatment groups on any of the patient and care measures reported. The study by Warner et al 2000 does not find any differences in any of the reported measures for behaviour/psychopathology, satisfaction, or psychiatric and healthcare use. ${ }^{19}$

\section{Rheumatoid arthritis}

Riemsma et al assessed a PHR intervention, as summarised in table 1. It has a high risk of bias. See table 2 for details about methodological limitations. Overall, there were differences in some of the eight process measures but not for any of the other measures. Visits to healthcare staff varied for both groups. There was a difference between the groups in the number of visits to general practitioners (intervention: 2.8, 2.6 and 1.9; control: 2.1, 2.1 and 2.6 (at baseline, 7 months and 19 months respectively); $\mathrm{p} \leq 0.05$ after Bonferroni correction). Physiotherapists in the PHR group had more favourable opinions of the coordination of care than those in the control group (this was measured on a five-point scale, where 1 is a negative, and 5 is a positive view; intervention: 3.63 ; control: 2.93 ; $\mathrm{p} \leq 0.05$ ). Continuity of care was also perceived by the physiotherapists to be better in the PHR group (intervention: 3.91; control: 3.25 ; $\mathrm{p} \leq 0.05$ ). There are no differences in opinion for the other healthcare professionals.

\section{Stroke}

The one study assessing a PHR intervention, as listed in table 1 , had a high risk of bias. See table 2 for the summary of the methodological limitations. The study is by Ayana et a ${ }^{21}$ and only reported data for $38 \%$ of recruited patients. Patient satisfaction was higher in the intervention group for three out of 16 satisfaction/opinion measures-'able to talk about problems' 
Table 2 Summary of quality assessment of studies

\begin{tabular}{|c|c|c|c|c|c|c|c|c|}
\hline \multirow[b]{2}{*}{$\begin{array}{l}\text { Chronic } \\
\text { disease area }\end{array}$} & \multirow[b]{2}{*}{ Study } & \multicolumn{7}{|c|}{ Quality assessment* } \\
\hline & & $\begin{array}{l}\text { Conflict of } \\
\text { interest }\end{array}$ & $\begin{array}{l}\text { Study } \\
\text { design }\end{array}$ & $\begin{array}{l}\text { Participant } \\
\text { selection }\end{array}$ & $\begin{array}{l}\text { Allocation } \\
\text { concealment } \\
\text { and blinding }\end{array}$ & $\begin{array}{l}\text { Data } \\
\text { collection }\end{array}$ & $\begin{array}{l}\text { Attributable to } \\
\text { intervention }\end{array}$ & $\begin{array}{l}\text { Appropriate } \\
\text { analysis }\end{array}$ \\
\hline \multirow[t]{4}{*}{ Diabetes } & Dijkstra et $a l^{8}$ & $?$ & ++ & ++ & + & + & + & + \\
\hline & Dijkstra et $a l^{9}$ & $?$ & ++ & ++ & + & + & + & + \\
\hline & Dijkstra et $a l^{10}$ & $?$ & + & ++ & $?$ & + & + & + \\
\hline & Simmons et $\left.a\right|^{11}$ & - & ++ & ++ & + & + & ++ & + \\
\hline \multirow[t]{6}{*}{ Oncology } & Cornbleet et $a l^{12}$ & - & ++ & ++ & - & - & + & + \\
\hline & Drury et $a l^{13}$ & $?$ & ++ & ++ & + & - & + & + \\
\hline & Lecouturier et $a l^{14}$ & $?$ & + & ++ & + & - & + & + \\
\hline & Williams et $a l^{15}$ & ++ & + & ++ & + & + & + & + \\
\hline & Finlay and $\mathrm{Wyatt}^{16}$ & $?$ & + & - & + & - & + & + \\
\hline & van Wersch et al ${ }^{17}$ & $?$ & + & + & - & - & + & - \\
\hline \multirow[t]{2}{*}{ Mental health } & Lester et $a l^{18}$ & $?$ & ++ & + & + & + & + & + \\
\hline & Warner et al ${ }^{19}$ & ++ & ++ & + & + & + & ++ & + \\
\hline $\begin{array}{l}\text { Rheumatoid } \\
\text { arthritis }\end{array}$ & Riemsma et $a l^{20}$ & $?$ & + & + & + & + & + & + \\
\hline Stroke & Ayana et $a l^{21}$ & $?$ & + & + & - & + & + & + \\
\hline Palliative care & Latimer et $a l^{22}$ & $?$ & ++ & + & $?$ & - & + & $?$ \\
\hline
\end{tabular}

*Quality assessment: ++ , criterion met; + , criterion partially met; - , criterion not met; ?, unclear from the information provided.

$(p=0.02)$ and 'happy with recovery' $(p=0.04)$, as well as feeling that they had made a complete recovery from the stroke $(p=0.01)$. However, in the 23 measures of communication, the intervention group patients were also less able to talk to staff or doctors about problems $(p=0.01)$. Apart from these, there are no differences between treatments.

\section{Palliative care}

The one study assessing a PHR intervention, as listed in table 1, had a high risk of bias. Methodological limitations are assessed in table 2. The study by Latimer et al 1998 did not find any differences in any of the five main outcome measures between intervention and control groups. ${ }^{22}$

\section{DISCUSSION}

As summarised in tables 2, 3, the effectiveness of the PHRs has not been convincingly demonstrated. Many of the studies have not included enough patients to show a difference between groups. The small number of differences identified between groups in some studies may result from biases introduced by weak study design rather than representing true effects of the PHR. There are also specific issues, which are discussed here.

\section{Population}

There is a lack of evidence supporting the implementation of a PHR in any chronic disease population. The PHR intervention and any auxiliary actions to support its implementation were different in each study. Although there are studies in six chronic disease populations representing a wide range of conditions, population characteristics might influence the effectiveness of a PHR in other illness groups. The utility of a PHR in the palliative care patient group is also questionable given the relatively short time frame for using such an intervention compared with other patient groups.

\section{Intervention and control}

This review is not evaluating the effectiveness of the individual components of a PHR, but we are aware of a study that did this in a primary care setting. ${ }^{23}$ The uniqueness of each study's intervention has made it difficult to evaluate the effectiveness of a PHR because not only were the PHR contents often unclear,

Table 3 Summary of results for each chronic disease group

\begin{tabular}{|c|c|c|}
\hline Chronic disease area & Results & Comments \\
\hline Diabetes & $\begin{array}{l}\text { The PHR does not offer significant advantages in terms of managing } \\
\text { overall diabetes treatment and lifestyle. Improvements in some } \\
\text { submeasures of process, behavioural, and physiological measures, } \\
\text { but most physiological, behavioural, communication and process } \\
\text { measures show no significant differences. }\end{array}$ & $\begin{array}{l}\text { The four studies assessing the interventions all } \\
\text { have a moderate to high risk of bias }\end{array}$ \\
\hline Oncology & $\begin{array}{l}\text { Differences are seen in a few outcome submeasures, but there is no } \\
\text { significant advantage of a PHR in terms of managing overall oncology } \\
\text { treatment and lifestyle }\end{array}$ & The six studies all have a high risk of bias \\
\hline Mental health & There are no statistically significant differences reported for any outcomes & The two studies both have a high risk of bias \\
\hline Rheumatoid arthritis & $\begin{array}{l}\text { There are small differences in some measures of behaviour and } \\
\text { participant opinions, but there is no significant difference between } \\
\text { treatments for the majority of measures of patient health status, various } \\
\text { physiological tests, behaviour and self efficacy }\end{array}$ & The one study has a high risk of bias \\
\hline Stroke & $\begin{array}{l}\text { There are no differences for the majority of the outcome measures. Only } \\
\text { three out of } 31 \text { measures of patient satisfaction and opinion were } \\
\text { significantly better with a PHR, and one measure was worse with a PHR. }\end{array}$ & The one included study has a high risk of bias \\
\hline Palliative care & There are no significant differences in any of the outcomes & The one included study has a high risk of bias \\
\hline
\end{tabular}


but some studies also combined the PHR with auxiliary components such as patient and/or healthcare staff education, coordinators and/or reminders (eg, posters in clinics). Other studies only provided a PHR with minimal written instructions on how to use the record. A 'standard' PHR is difficult to pinpoint because there is no agreed design for any chronic disease patient group.

The implementation time of the PHRs was usually between 3 and 6 months. It is unclear if the implementation times were adequate in these studies.

Some suggest that to improve PHR implementation, changes are necessary in the form and content of the PHR, attitudes of staff to utilise the PHR and attitudes of patients to be proactive in their own care. ${ }^{17}$ This raises further questions about what auxiliary tools can be effectively combined with a PHR to improve its effectiveness, which was unclear from these studies.

Blinding of the intervention is important but not always done properly. A method used to blind the participants to the PHR was by using 'sham' or control records that were unstructured. However, the extent of the control record actually being a control is flawed. The most minimally structured PHR used as intervention in one study could be equivalent to a control record in another study. Also, patients may have their own medical 'diaries' to record their treatments, medicines, appointments and other details. There is nothing preventing this or letting us know if patients were doing this in the control groups.

\section{Outcomes}

Many of the reported outcomes were patient and healthcare staff opinions and attitudes towards the intervention. Caution is needed for interpreting these results due to high levels of subjectivity and risk of bias. Thirteen of the 14 studies report that patients and staff usually perceived that there was no clear benefit of using the intervention compared with usual care. $^{8}{ }^{10-16} 18-22$ Feedback from staff in two studies indicates uncertainty in using the PHR due to an already heavy work load. ${ }^{1521}$ The use of additional organisational support, such as coordinators, in some studies was an attempt to address this barrier to implementation. However, this level of support is unlikely to be available in most clinical environments. The elements of staff buy-in and organisational support might be influential, but the results are unclear about this. In the rheumatoid arthritis population, even though the intervention enhanced communication between staff, it did not enhance the majority of other outcomes. ${ }^{20}$ Many of the included studies showed that utilisation of the PHR by patients was low. The low level of patient adherence to the intervention is a reason to cautiously interpret the reported outcomes.

\section{Implications for further research}

There is currently insufficient information to know what the components of a PHR should be, how it should be delivered, what support should be provided and what education and training are required for staff. A PHR needs to be developed, piloted and revised prior to implementation, addressing the issues discussed here, with proper RCTs.

\section{CONCLUSIONS}

From the 14 studies included in this review in six chronic disease groups, there is no high-quality evidence showing the effectiveness of PHRs. These poor quality studies do not show a clear benefit of implementing a PHR. More high-quality studies are required in order to make firm conclusions about the effectiveness of PHRs in chronic disease populations.

\section{What is already known about this topic}

PHRs aim to improve communication between patients and clinicians, and management of healthcare tasks. These have been used in health areas such as child health and maternal health. There are conflicting opinions on the effectiveness of PHRs.

\section{What this study adds}

Given the levels of bias described, these studies have not convincingly demonstrated a benefit of PHRs in this patient group.

Acknowledgements Kelly Allen and Claire Harris for their comments on the appraisals of the studies.

Competing interests None.

\section{Patient consent Obtained.}

Contributors $\mathrm{CJ}$ and $\mathrm{CH}$ conceived the study and provided clinical interpretation. HK is guarantor. HK and TT designed the protocol, analysed and interpreted the data. All authors approved the final manuscript. HK collected the data. HK and TT draughted the manuscript. HK and $\Pi$ critically revised the manuscript for important intellectual content. All authors approved the final version to be published.

Provenance and peer review Not commissioned; not externally peer reviewed.

\section{REFERENCES}

1. Gysels M, Richardson A, Higginson IJ. Does the patient-held record improve continuity and related outcomes in cancer care: a systematic review. Health Expect 2007:10:75-91.

2. Saffin K. Parent-held records of child development. Nurs Times 1991;87 54-5.

3. Cohen S, Gitterman B, Baron A, et al. Improving adherence with preventive pediatric care guidelines through the use of a parent-held child health record [abstract]. AHSR FHSR Annu Meet Abstr Book 1994;11:113.

4. Grovdal L, Grimsmo A, Nilsen TIL. Parent-held child health records do not improve care: a randomized controlled trial in Norway. Scand J Prim Health Care 2006;24:186-90.

5. Dickey LL, Petitti D. Assessment of a patient-held minirecord for adult health maintenance. J Fam Pract 1990;31:431-8.

6. Dickey LL, Petitti D. A patient-held minirecord to promote adult preventive care. J Fam Pract 1992;34:457-63.

7. Briss P, Rodewald L, Hinman A, et al. The task force on community preventive services: reviews of evidence regarding interventions to improve vaccination coverage in children, adolescents, and adults. Am J Prev Med 2000;18:97-140.

8. Dijkstra RF, Braspenning JCC, Huijsmans Z, et al. Grol RPTM: introduction of diabetes passports involving both patients and professionals to improve hospital outpatient diabetes care. Diabetes Res Clin Pract 2005;68:126-4.

9. Dijkstra RF, Niessen LW, Braspenning JCC, et al. Patient-centred and professionaldirected implementation strategies for diabetes guidelines: A cluster-randomized trial-based cost-effectiveness analysis. Diabetic Medicine 2006;23:164-70.

10. Dijkstra R, Braspenning J, Grol R. Implementing diabetes passports to focus practice reorganization on improving diabetes care. Int $\mathrm{J}$ Qual Health Care 2008:20:72-7.

11. Simmons D, Gamble GD, Foote $S$, et al. The New Zealand diabetes passport study: a randomized controlled trial of the impact of a diabetes passport on risk factors for diabetes-related complications. Diabetic Medicine 2004;21:214-17.

12. Cornbleet MA, Campbell P, Murray $S$, et al. Patient-held records in cancer and palliative care: a randomized, prospective trial. Palliat Med 2002;16:205-12.

13. Drury M, Yudkin P, Harcourt J, et al. Patients with cancer holding their own records: a randomised controlled trial. Br J Gen Pract 2000;50:105-10.

14. Lecouturier J, Crack L, Mannix K, et al. Evaluation of a patient-held record for patients with cancer. Eur J Cancer Care 2002;11:114-21.

15. Williams JG, Cheung WY, Chetwynd N, et al. Pragmatic randomised trial to evaluate the use of patient held records for the continuing care of patients with cancer. Qual Health Care 2001;10:159-65. 
16. Finlay IG, Wyatt P. Randomised cross-over study of patient-held records in oncology and palliative care. Lancet 1999;353:558-9.

17. van Wersch $\mathbf{A}$, de Boer MF, de Jong $P$, et al. Continuity of information in cancer care: evaluation of a logbook. Patient Educ Couns 1997;31:223-36.

18. Lester $\mathbf{H}$, Allan T, Wilson $\mathrm{S}$, et al. A cluster randomised controlled trial of patientheld medical records for people with schizophrenia receiving shared care. $\mathrm{Br} J \mathrm{Gen}$ Pract 2003;53:197-203.

19. Warner JP, King M, Blizard R, et al. Patient-held shared care records for individuals with mental illness: randomised controlled evaluation. $\mathrm{Br} \mathrm{J}$ Med Psychol 2000;177:319-24.
20. Riemsma RP, Taal E, Brus HL, et al. Coordinated individual education with an arthritis passport for patients with rheumatoid arthritis. Arthritis Care Res 1997; 10:238-49.

21. Ayana M, Pound P, Lampe F, et al. Improving stroke patients' care: a patient held record is not enough. BMC Health Serv Res 2001;1:1-6.

22. Latimer EJ, Crabb MR, Roberts JG, et al. The patient care travelling record in palliative care: effectiveness and efficiency. J Pain Symptom Manage 1998; 16:41-51.

23. Giglio RJ, Papazian B. Acceptance and use of patient-carried health records. Med Care 1986;24:1084-92. 